OPEN

SUBJECT AREAS: BIOLOGICAL METAMORPHOSIS

ICHTHYOLOGY

Received

12 August 2014

Accepted

16 December 2014

Published

19 January 2015

Correspondence and requests for materials should be addressed to

A.I. (atsuo@frontier. kyoto-u.ac.jp)

\title{
Prenatal regression of the trophotaenial placenta in a viviparous fish, Xenotoca eiseni
}

\author{
Atsuo lida ${ }^{1}$, Toshiyuki Nishimaki \& Atsuko Sehara-Fujisawa ${ }^{1}$
}

\begin{abstract}
'Department of Growth Regulation, Institute for Frontier Medical Sciences, Kyoto University, Kawahara-cho 53, Shogo-in, Kyoto 606-8507, Japan, ${ }^{2}$ Department of Anatomy, Kitasato University School of Medicine 1-15-1 Kitasato, Sagamihara Kanagawa 228 8555 , Japan.
\end{abstract}

The trophotaenial placenta is a branching, ribbon-like structure that extends from the perianal region of the embryo in viviparous teleost fishes belonging to the family Goodeidae. It is a hindgut-derived pseudoplacenta, which contributes to absorbing maternal nutrients during the prenatal stage. The trophotaeniae are known to reduce at birth; however, no previous study has evaluated the removal mechanisms. We report here the analysis of the trophotaeniae using the goodeid fish species Xenotoca eiseni. The $X$. eiseni trophotaenia consists of an epidermal cell layer, mesenchyme, vasculature, and circulating erythrocytes. The trophotaeniae had preliminary regressed when the embryo was born. Immunohistochemistry indicated that caspase3-activated cells with fragmented nuclei are present in the regressed processes of the fry immediately after birth, but not in the vasculature and blood cells. This finding suggests that the trophotaenia is rapidly resorbed by apoptosis in the last phase of the pregnancy and that its circulatory pathway is maintained. Such prenatal regression of pseudoplacentae has not been reported in other viviparous vertebrates. On the other hand, similar apoptotic remodeling in the gut has been reported in amphibians, which is regulated by thyroid hormone. Thus, apoptotic regression of the trophotaeniae might occur in a manner similar to amphibian metamorphosis.

$\mathrm{V}$ iviparous animals are widely distributed in the extant vertebrates ${ }^{1}$. In mammals, all species, excluding the monotremes, show embryonic growth and development within the female body, supported by the provision of maternally derived nutrients. For nutrient absorption, mammals have a placenta and umbilical cords fused to the mother's body. In the case of non-mammalian vertebrates, the viviparous reptile Trachylepis ivensi has a presumed homolog to the mammalian placenta and umbilical cord ${ }^{2}$. In addition, some viviparous cartilaginous fishes possess a yolk sac-derived pseudoplacenta that forms during embryonic development in the mother's body ${ }^{3}$. These diverse viviparous reproduction systems are considered to have evolved independently in vertebrates.

Over 500 species of teleost fish have been identified as viviparous, and in some species, the embryo weight increases during pregnancy ${ }^{4}$. Thus, these fishes likely possess the specific machinery required to absorb maternally derived nutrients. In particular, the order Cyprinodontiformes includes approximately 170 viviparous species $^{5-8}$. In this study, we focused on a viviparous teleost species that belongs to the family Goodeidae (Xenotoca eiseni). X. eiseni is distributed in the lakes and rivers of the Central Plateau of Mexico and is known to have a unique structure, the "trophotaenial placenta," which is a pseudoplacenta that functions to absorb the maternally derived nutrients ${ }^{9}$. Their eggs hatch in the ovary of the mother's body, where the embryos develop until birth. This absorption of nutrients via the trophotaeniae allows for the X. eiseni offspring to be born at a more advanced stage relative to that observed in oviparous and ovoviviparous fishes. A previous study showed that all viviparous species belonging to the family Goodeidae, excluding Ataeniobius toweri, possess a trophotaenial placenta during their development ${ }^{10,11}$. These trophotaeniae were observed as a hindgut-derived ribbon-like structure that extends from the perianal region of the embryo. Given that the trophotaenia is not fused to the mother's body, these goodeid embryos likely receive the maternally derived nutrients provided from the ovarian lumen in a secreted form ${ }^{12,13}$. Therefore, this structure is not a homologous organ of the mammalian placenta and umbilical cord, neither anatomically nor functionally.

The trophotaenial placenta is only transiently required for absorption of the maternally derived nutrients during development. Since the offspring can ingest food orally soon after birth, the trophotaeniae become 
unnecessary. In fact, the trophotaeniae of goodeid embryos show preliminary regression at birth and then disappear within a few days $^{5,14}$. Thus, the removal machinery for the trophotaeniae begins to function at the prenatal stage. However, no previous studies have elucidated the specific regression mechanisms of the trophotaeniae. Therefore, we used $X$. eisen $i$ as a model viviparous goodeid species to analyze the trophotaenial placenta in detail ${ }^{14,15}$.

\section{Results}

Observation of the trophotaenial placenta in the developing embryo of $X$. eiseni. We purchased $X$. eiseni from a commercial supplier, and the fish were bred in our fish facility. The pregnancy duration of $X$. eiseni was approximately five weeks (34-39 days) under our breeding conditions. The embryos were obtained from pregnant $X$. eiseni females at the $2 \mathrm{nd}, 3 \mathrm{rd}$, and 4 th weeks after mating (Fig. 1a-1c). They showed different degrees of trophotaeniae elongation from the perianal region, depending on the stage of embryonic development (Fig. 1d-1f). The trophotaeniae were not fused to the maternal tissues, and no decidual-like structure was observed on the ovarian lumen.

Histological analyses of sections of the 4th-week embryos stained using hematoxylin-eosin (HE) indicated that the trophotaeniae have a complicated structure, consisting of an epidermal cell layer, mesenchyme, vasculature, and blood cells (Fig. 1g). The processes were continuous with the gastrointestinal submucosa or epidermis of the fry (Supplementary Fig. 1), similar to a previous description of this fish family ${ }^{5}$. However, the precise origin of each component in the trophotaeniae could not be identified in this study. Fluorescent microscopy revealed that the epidermal cell layer could be labeled using a fluorescent-conjugated phalloidin marker, which was found to be associated to filamentous actins. The fibronectin-rich mesenchyme was surrounded by the epithelial-like component. Globin-pos- itive erythrocytes were detected in the lumen of the phalloidinassociated vasculature walls (Fig. $1 \mathrm{~h}-1 \mathrm{j}$ ). Live observations indicated that the blood cells and the plasma component were circulating in the processes throughout the embryonic body (Supplementary Movie 1). These observations indicated that the $X$. eiseni embryo possesses a typical trophotaenial placenta that is similar to that of other viviparous species belonging to the family Goodeidae $e^{12,13}$.

Prenatal regression of the trophotaenial placenta in the fry. We observed shrunken processes at the perianal region of the fry immediately after birth, which were considered to be residues of the trophotaeniae (Fig. 2a \& 2b). Histological observations showed that the epidermal cell layer and mesenchymal structures were lost in the regressed processes. The vasculature showed a snaking pattern, and blood cells were observed in the vascular lumen (Fig. $2 c \&$ Fig. 3b). The developmental stage of the fry was estimated to be at around the 5th week post-fertilization. The 4th-week embryos still had complete processes with no signs of regression (Fig. 1f \& $1 \mathrm{~g}$ ). These observations suggested that the trophotaeniae undergo rapid regression in the mother's body at the last phase of pregnancy. Because of the important role that the trophotaenial placenta plays for uptake of maternally derived nutrients during embryonic development in the mother's body, prenatal regression should be controlled under a firm regulation mechanism.

Activation of the apoptosis pathway in the regression process. Apoptosis, a process of programmed cell death involving nucleus fragmentation, widely contributes to disease development, physiological tissue turnover, and morphogenesis during development ${ }^{16,17}$. Caspase 3 activation is a major marker of apoptosis-related cell death $^{18}$. To determine the role of apoptosis in the prenatal regression of the trophotaeniae, the regressed processes were stained using
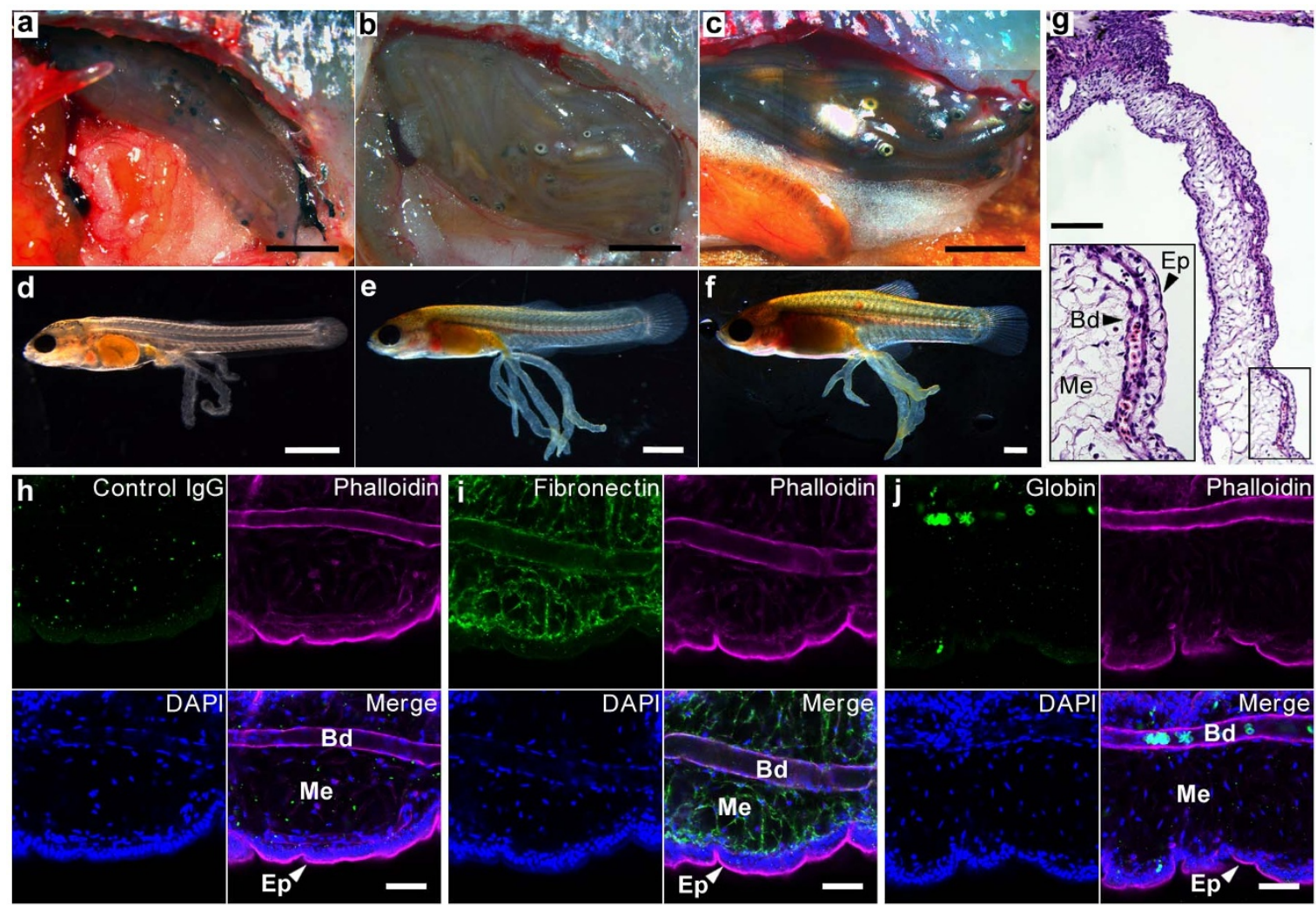

Figure $1 \mid$ Histological analysis of the trophotaenial placenta. (a-c). The ovaries of pregnant females at the 2nd (a), 3rd (b), and 4th week (c) postmating. Scale bar: $5 \mathrm{~mm}$. (d-f). The embryo extracted from the ovaries at the 2nd (d), 3rd (e), and 4th week (f). Scale bar: $1 \mathrm{~mm}$. (g). Hematoxylin-eosinstained section of the trophotaeniae of a 4th-week embryo. Scale bar: $100 \mu \mathrm{m}$. $(\mathrm{h}-\mathrm{j})$. Fluorescent immunochemistry to visualize the structure of the trophotaeniae in the 4th-week embryo. Scale bar: $50 \mu \mathrm{m}$. Bd, blood vessel; Ep, epidermal cell layer; Me, mesenchyme. 

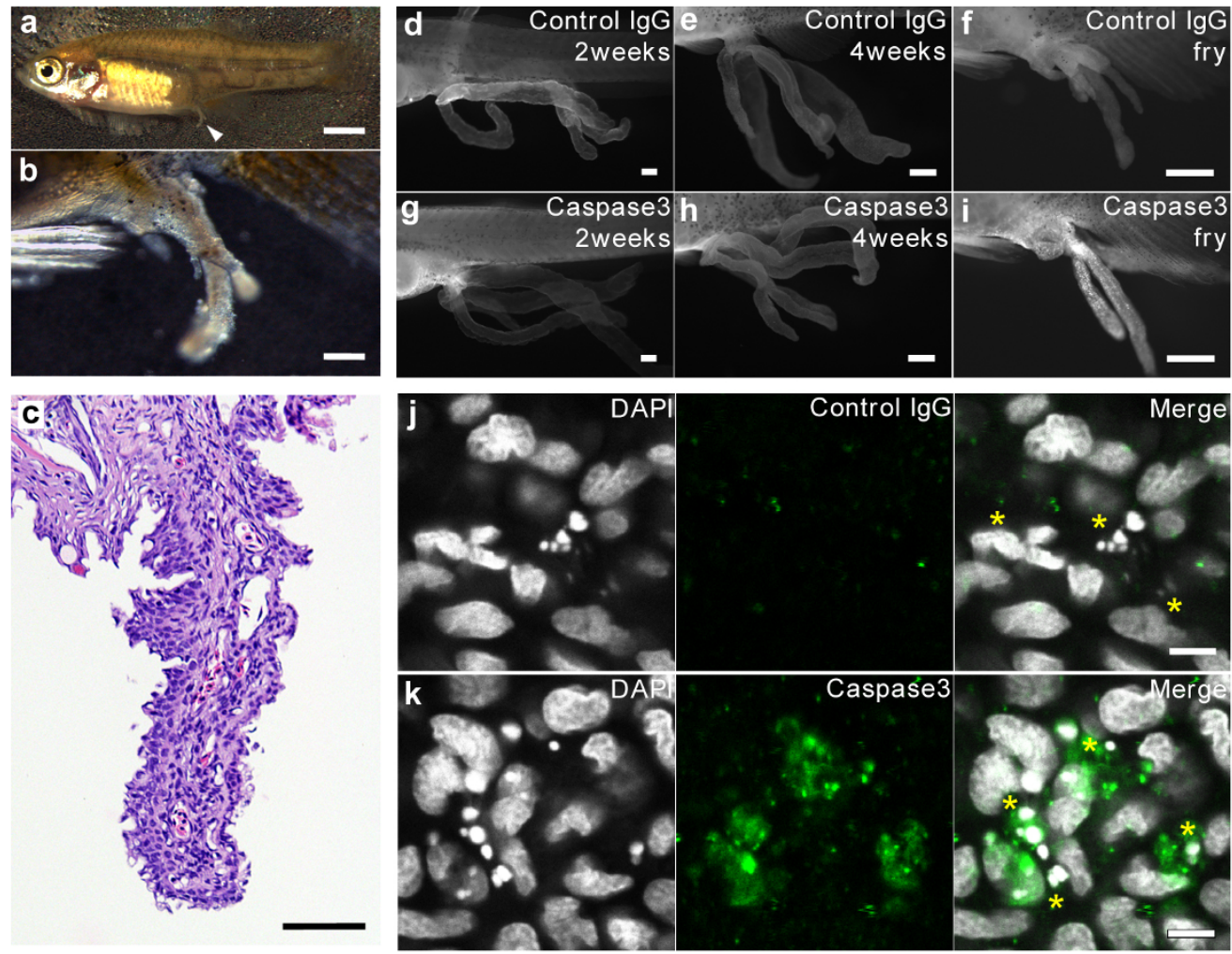

Figure $2 \mid$ Programmed cell death in the regressed processes. (a-b). Full image of a fry immediately after birth (a) and an enlarged image of the regressed process, a vestige of the trophotaenia in the fry (b). Scale bar: $2 \mathrm{~mm}$ (a) and $200 \mu \mathrm{m}$ (b). (c). Hematoxylin-eosin-stained section of the regressed processes in the fry. Scale bar: $50 \mu \mathrm{m}$. (d-i). Fluorescent immunochemistry to detect the apoptotic cells in the trophotaenial placenta (2nd and 4 th weeks) and the regressed processes (fry). Scale bar: $500 \mu \mathrm{m}$. ( $\mathrm{j}-\mathrm{k}$ ). Confocal microscopy of fluorescent immunochemistry to detect the apoptotic cells in the regressed processes. The asterisks indicate the apoptotic cells defined by fragmented nuclei. Scale bar: $5 \mu \mathrm{m}$.

anti-activated caspase 3 antibody and 4'6-diamidino-2-phenylindole (DAPI). Fluorescent microscopy showed positive active-caspase 3 antibody labeling, indicating the presence of some apoptotic cells in the regressed processes of the fry (Fig. $2 \mathrm{f} \& 2 \mathrm{i}$ ). These cells were not detected in the elongated trophotaeniae of the 2 nd- or 4 th-week embryos (Fig. 2d, 2e, 2g, \& 2h). In addition, confocal microscopy revealed that the active-caspase 3 signals were merged with fragmented nuclei in the regressed processes (Fig. 2j \& $2 \mathrm{k}$ ). These observations indicated that the trophotaenial placenta of $X$. eisen $i$ prenatally regresses by a process of apoptotic cell death (Fig. 4a).

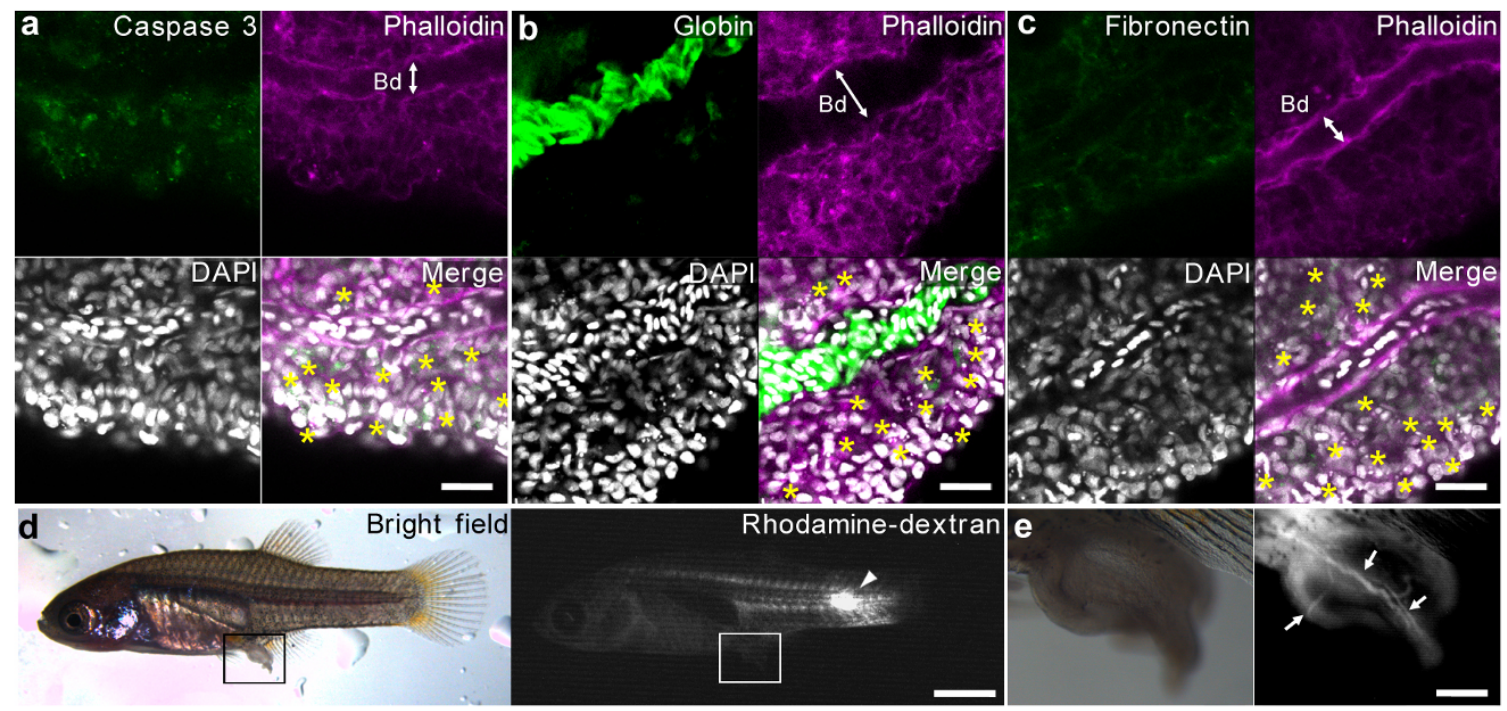

Figure 3 Maintenance of the circulatory pathway during the regression. (a-c). Confocal microscopy of the fluorescent immunochemistry to visualize the structure of the regressed processes. The asterisks indicate the apoptotic cells defined by fragmented nuclei, which are merged with the activecaspase3 in (a). Scale bar: $20 \mu \mathrm{m}$. (d). Micro-angiography of the vascular network of the regressed processes. The arrowheads indicate the dextraninjected site. Scale bar: $2 \mathrm{~mm}$. (e). Enlarged image of the regressed processes. The arrows indicate the intravascular lumen visualized by dextran. Scale bar: $200 \mu \mathrm{m}$. Bd, blood vessel. 


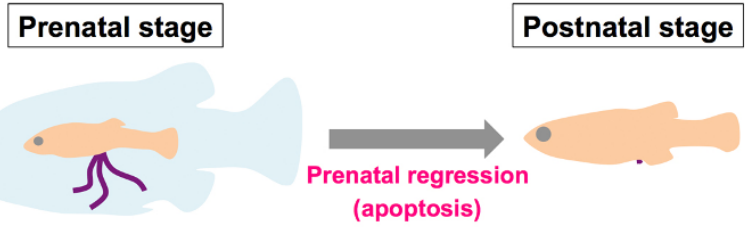

b

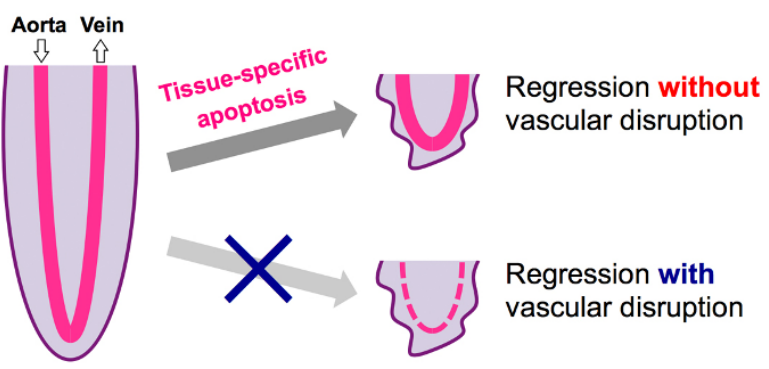

Figure $4 \mid$ Prenatal regression in the trophotaenial placenta of Xenotoca eiseni. (a). Schematic illustration summarizing the model of trophotaenial placenta changes during development. The trophotaeniae (purple) begin to disappear at the last stage of pregnancy before birth. This "prenatal regression" process is regulated by apoptosis. (b). Schematic illustration summarizing the tissue-specific regression. The trophotaenia prenatally regressed without disruption of the circulatory pathway (vasculature in the trophotaenia, magenta).

Tissue-specific apoptosis activation during prenatal regression. Confocal microscopic observations indicated the presence of apoptotic cells, as determined by active-caspase 3 staining and fragmented nuclei, in specific tissues of the regressed processes: the epidermal cell layer and mesenchyme (Fig. 3a-3c). In addition, the fibronectin-associated mesenchyme showed irregular shapes and there was no phalloidin-labeled epidermal cell layer in the regressed processes (compare Fig. $3 c$ to Fig. 1i). These results suggested that the epidermal and mesenchymal cells might receive the apoptosis signal. In contrast, the vasculature and blood cells appear to have low sensitivity or to be resistant to these signals of apoptotic cell death. These results suggested that the blood circulation system of the trophotaeniae survives and maintains its function during the regression process. To verify the function of the circulatory system in the postnatal stage, fluorescent-conjugated dextran was injected into the cardinal vein of the fry. The fluorescence signal demonstrated a vascular network not only in the body but also in the regressed processes (Fig. 3d \& 3e). Microangiography indicated that a closed vascular network from the body to the trophotaeniae was maintained during the regression process. In addition, live observations indicated that the intravascular blood cells circulated through the regressed processes in a manner similar to that observed during the prenatal stage (Supplementary Movie 2 \& 3 ). These results confirmed that the circulatory pathway in the trophotaenial placenta is indeed maintained during the regression phase (Fig. 4b).

\section{Discussion}

In this study, we elucidated the apoptotic regression process for the postnatal removal of the trophotaenial placenta using the goodeid fish species $X$. eiseni. To the best of our knowledge, there are no previous reports about such apoptotic regression in placentae or pseudoplacentae that function to absorb maternally derived nutrients during the development of viviparous vertebrates. In humans, a few apoptotic cells are also detectable in the placenta throughout pregnancy ${ }^{19}$. However, there are no reports of any positive roles of apoptosis in the fetal development of mammals, and an increase in apoptotic cells instead indicates a pathological process that could result in placental disorders ${ }^{20}$. In the case of amphibians, an apopto- sis-regulated mechanism of tissue/organ remodeling occurs in the tail, brain, and intestine during metamorphosis ${ }^{21}$. As described previously, the trophotaenial placenta of $X$. eiseni is derived from the hindgut. Thus, the apoptotic regression and morphological changes occurring in the goodeid embryo might represent a similar mechanism to that of amphibian metamorphosis.

We did not identify the specific upstream pathway responsible for apoptosis activation. Previous studies reported that apoptosis is induced by an intrinsic and/or extrinsic pathway ${ }^{22}$. One possibility is that an extrinsic signal is transmitted from the mother to the embryo. In this scenario, a direct trigger for apoptosis activation is provided in the form of maternal elements. Alternatively, the embryo might autonomously regulate the apoptosis by an intrinsic and/or extrinsic pathway. One such candidate is the thyroid hormone (TH), which contributes to amphibian metamorphosis and induces apoptosis in the larval intestine ${ }^{21}$. $\mathrm{TH}$ and its receptors are conserved in teleosts and are expressed in the embryonic stage and contribute to developmental changes in the gut and other organs $s^{23,24}$. Thus, these molecules are potent candidates as embryo-autonomous factors of apoptosis induction. Supporting the hypothesis that the embryo autonomously induces apoptosis, a 4 th-week embryo surgically extracted from the mother's body was found to initiate the apoptosis-dependent regression of the trophotaenial placenta (Supplementary Figure 2). However, this observation does not rule out the possibility of a maternal contribution. Further studies are required to determine the factors that regulate this apoptosis and whether any maternal factors are involved.

Our results also indicate that apoptotic regulation is a tissue-specific phenomenon. It could be a mechanism to prevent a hemorrhage during the regression process, which would prevent the possibility of necrotic cell death or starvation due to arrest of the circulation and nutrient transport. Another possibility is that the surviving cells contribute to vascular rearrangement during the regression process. A previous study showed that vascular networks at the perianal region of another goodeid fish, Skiffia bilineata, were dramatically rearranged during the perinatal stage $\mathrm{e}^{25}$. Thus, our findings suggest that prenatal regression involves not only tissue-specific apoptotic disruption of the unnecessary processes but also serves to help remodel the vascular networks to form an alternate circulatory pathway at the perianal region. Therefore, determining the specific regulatory mechanism involved in the initiation of trophotaeniae regression is an important topic to help further understand the viviparous machinery of the goodeid fish.

\section{Experimental Procedures}

Animal experiments. This study was approved by the ethics review boards for animal experiments of Kyoto University. We sacrificed live animals in minimal numbers under anesthesia according to the institutional guidelines.

Fish breeding. Xenotoca eiseni was purchased from Meito Suien Co., Ltd. (Nagoyacity, Japan). Adult fish were maintained in freshwater at $27^{\circ} \mathrm{C}$ under a $14: 10$-h lightdark photoperiod cycle. Fish were bred in a mass-mating design, and approximately 50 fish were maintained for this study. The juveniles were fed live brine shrimp larvae and Hikari Rabo 450 fish food (Kyorin Co., Ltd.; Himeji-city, Japan), and the adults were fed Hikari Crest Micro Pellets (Kyorin). To accurately track the pregnancy period, the laboratory-born fish were crossed in a pair-mating design, and the mating behavior was recorded.

Embryo extraction. Pregnant females were anesthetized using tricaine on ice, following which the embryos were surgically extracted. In this study, we dissected approximately 20 pregnant females, and extracted 20-40 embryos in each operation. In order to collect the fry immediately after birth, we checked the females in late pregnancy every day. Approximately $10-30$ live fry $(\sim 15 \mathrm{~mm}$ in length) were obtained from each delivery. Some fry were dead at birth. Microscopy observations were performed with a Leica M205C microscope (Leica Microsystems; Mannheim, Germany).

Histology. Fish samples were fixed with Davidson's fixative solution (33\% ethanol, $8 \%$ formaldehyde, and $11 \%$ acetic acid) at room temperature. Preparation of paraffin sections and HE staining were performed by following a standard protocol in our laboratory, or by Biopathology Institute Co., Ltd. (Kunisaki-city, Japan). 
Immunohistochemistry. Fish samples were fixed in $4 \%$ paraformaldehyde/ phosphate-buffered saline (PBS) at $4{ }^{\circ} \mathrm{C}$ overnight. Fixed samples were permeabilized using $0.5 \%$ TritonX-100/PBS at room temperature for $30 \mathrm{~min}$, and then treated with Blocking-One solution (Nacalai Tesque) at room temperature for $1 \mathrm{~h}$. Antiactivated-caspase3 (rabbit, Sigma-Aldrich), anti-fibronectin (rabbit, Sigma-Aldrich), and anti-ey-globin (rabbit, a gift from Dr. T. Atsumi, RIKEN; Wako-city, Japan) were used as the primary antibodies ${ }^{26}$. Each antibody was used in $1: 500$ dilution with Blocking-One solution. Samples were reacted with primary antibodies at $4{ }^{\circ} \mathrm{C}$ overnight. An Alexa-488 secondary antibody (Life Technologies) was used at 1:500 dilution in $0.1 \%$ Tween-20/PBS with Alexa Fluor ${ }^{\circledR} 546$ Phalloidin (Life Technologies) and DAPI (Sigma-Aldrich). Samples were treated in the secondary antibody solution at $4^{\circ} \mathrm{C}$ overnight. Microscopic observation was performed using Leica MZ16FA, Leica TCS SP5, and Leica TCS SP8 microscopes (Leica Microsystems).

Micro-angiography. Collected fry were anesthetized on ice. A rhodamineconjugated dextran/PBS solution was injected into the caudal vein of the fry by using a pneumatic microinjection system (NARISHIGE). Microscopy observations were performed with a Leica M205C microscope.

1. Blackburn, D. G. Evolution of vertebrate viviparity and specializations for fetal nutrition: A quantitative and qualitative analysis. J. Morphol.; DOI: 10.1002/ jmor.20272. (2014)

2. Blackburn, D. G. \& Flemming, A. F. Invasive implantation and intimate placental associations in a placentotrophic African lizard, Trachylepis ivensi (Scincidae). J. Morphol. 273, 137-159 (2012).

3. Castro, J. I. Observations on the reproductive cycles of some viviparous North American sharks. Aqua 15, 4-15 (2009).

4. Wourms, J. P. Viviparity: the maternal-fetal relationship in fishes. Amer. Zool. 21, 473-515 (1981).

5. Turner, C. L. Viviparity superimposed upon ovo-viviparity in the goodeidae, a family of cyprinodont teleost fishes of the Mexican Plateau. J. Morphol. 55 207-251 (1933).

6. Turner, C. L. Adaptations for viviparity in embryos and ovary of Anableps anableps. J. Morphol. 62, 323-349 (1938).

7. Turner, C. L. Pseudoamnion, pseudochorion, and follicular pseudoplacenta in poeciliid fishes. J. Morphol. 67, 59-89 (1940).

8. Turner, C. L. Adaptations for viviparity in jenynsiid fishes. J. Morphol. 67, 291-297 (1940).

9. Lombardi, J. \& Wourms, J. P. The trophotaenial placenta of a viviparous goodeid fish. II. Ultrastructure of trophotaeniae, the embryonic component. J. Morphol. 184, 293-309 (1985)

10. Turner, C. L. The trophotaeniae of the goodeidae, a family of viviparous cyprinodont fishes. J. Morphol. 61, 495-523 (1937).

11. Schindler, J. F. \& Hamlett, W. C. Maternal-embryonic relations in viviparous teleosts. J. Exp. Zool. 266, 378-393 (1993).

12. Lombardi, J. \& Wourms, J. P. The trophotaenial placenta of a viviparous goodeid fish. III: Protein uptake by trophotaeniae, the embryonic component. J. Exp. Zool. 236, 165-179 (1985)

13. Schindler, J. F. Structure and function of placental exchange surfaces in goodeid fishes (Teleostei: Atheriniformes). J. Morphol. DOI: 10.1002/jmor.20292. (2014).

14. Mendoza, G. The ovary and anal processes of "Characodon"eiseni, a viviparous cyprinodont teleost from Mexico. Biol. Bull. 129, 303-315 (1965).

15. Mendoza, G. The fine structure of an absorptive epithelium in a viviparous teleost. J. Morphol. 136, 109-130 (1972).
16. Jacobson, M. D., Weil, M. \& Raff, M. C. Programmed cell death in animal development. Cell 88, 347-354 (1997).

17. Yaron, F. \& Hermann, S. Programmed cell death in animal development and disease. Cell 147, 742-758 (2011).

18. Sakahira, H., Enari, M. \& Nagata, S. Cleavage of CAD inhibitor in CAD activation and DNA degradation during apoptosis. Nature 391, 96-99 (1998).

19. Smith, S. C., Baker, P. N. \& Symonds, E. M. Placental apoptosis in normal human pregnancy. Am. J. Obstet. Gynecol. 177, 57-65 (1997).

20. Sharp, A. N., Heazell, A. E., Crocker, I. P. \& Mor, G. Placental apoptosis in health and disease. Am. J. Reprod. Immunol. 64, 159-169 (2010).

21. Ishizuya-Oka, A., Hasebe, T. \& Shi, Y. B. Apoptosis in amphibian organs during metamorphosis. Apoptosis 15, 350-364 (2010).

22. Fulda, S. \& Debatin, K. M. Extrinsic versus intrinsic apoptosis pathways in anticancer chemotherapy. Oncogene 25, 4798-4811 (2006).

23. Power, D. M. et al. Thyroid hormones in growth and development of fish. Comp. Biochem. Physiol. C Pharmacol. Toxicol. Endocrinol. 130, 447-459 (2001).

24. Specker, J. L. Preadaptive role of thyroid hormones in larval and juvenile salmon: growth, the gut and evolutionary considerations. Amer. Zool. 28, 337-349 (1988).

25. Mendoza, G. Structural and vascular changes accompanying the resorption of the proctodaeal processes after birth in the embryos of the Goodeidae, a family of viviparous fishes. J. Morphol. 61, 95-125 (1937).

26. Miwa, Y., Atsumi, T., Imai, N. \& Ikawa, Y. Primitive erythropoiesis of mouse teratocarcinoma stem cells PCC3/A/1 in serum-free medium. Development 111, 543-549 (1991).

\section{Acknowledgments}

We thank Nobutoshi Mizuno, Tomoharu Watanabe, Yukimasa Onabe, and Yuda Tokumasu for helpful discussions. Yukiko Ogino kindly provided comments on previous drafts. Some of the microscopy observations were performed in the Kyoto University-Leica Imaging Center (CiLKy). This work was supported by the Kyoto University Research Administration Office (KURA) and the Japan Society for the Promotion of Science KAKENHI Grant (Number 24657161 to A.I.).

\section{Author contributions}

A.I. designed the experiments. A.I. and T.N. performed the experiments. A.I. and A.S.F. wrote the paper.

\section{Additional information}

Supplementary information accompanies this paper at http://www.nature.com/ scientificreports

Competing financial interests: The authors declare no competing financial interests.

How to cite this article: Iida, A., Nishimaki, T. \& Sehara-Fujisawa, A. Prenatal regression of the trophotaenial placenta in a viviparous fish, Xenotoca eiseni. Sci. Rep. 5, 7855; DOI:10.1038/srep07855 (2015).

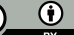

This work is licensed under a Creative Commons Attribution 4.0 International License. The images or other third party material in this article are included in the article's Creative Commons license, unless indicated otherwise in the credit line; if the material is not included under the Creative Commons license, users will need to obtain permission from the license holder in order to reproduce the material. To view a copy of this license, visit http://creativecommons.org/licenses/by/4.0/ 